\title{
Alternative Formulations for Incorporating Lateral Boundary Data into Limited-Area Models
}

\author{
MARTINA TUDOR \\ Croatian Meteorological and Hydrological Service, Zagreb, Croatia \\ PIET TERMONIA \\ Royal Meteorological Institute of Belgium, Brussels, Belgium
}

(Manuscript received 3 August 2009, in final form 11 January 2010)

\begin{abstract}
Limited-area models (LAMs) use higher resolutions and more advanced parameterizations of physical processes than global numerical weather prediction models, but suffer from one additional source of error-the lateral boundary condition (LBC). The large-scale model passes the information on its fields to the LAM only over the narrow coupling zone at discrete times separated by a coupling interval of several hours. The LBC temporal resolution can be lower than the time necessary for a particular meteorological feature to cross the boundary. A LAM user who depends on LBC data acquired from an independent prior analysis or parent model run can find that usual schemes for temporal interpolation of large-scale data provide LBC data of inadequate quality. The problem of a quickly moving depression that is not recognized by the operationally used gridpoint coupling scheme is examined using a simple one-dimensional model. A spectral method for nesting a LAM in a larger-scale model is implemented and tested. Results for a traditional flow-relaxation scheme combined with temporal interpolation in spectral space are also presented.
\end{abstract}

\section{Introduction}

Limited-area models (LAMs) are used as an alternative to global numerical weather prediction (NWP) models for a wide variety of research and operational forecast applications. Particularly LAMs are subject to different sources of forecast error: the parameterizations of physical processes, the initial conditions, the numerical algorithms, and surface forcing. These also affect various global NWP models, but LAMs have one additional source of error related to their lateral boundary conditions (LBCs). The most popular scheme for LBC treatment is the one proposed by Davies (1976), used almost exclusively for one-way coupled operational LAMs (McDonald 1999). There are problems that are linked with the nature of various lateral boundary schemes (Davies 1983), but LBC problems can also be of a different source (e.g., the quality of the large-scale data). An overview of the

Corresponding author address: Martina Tudor, Croatian Meteorological and Hydrological Service, Gric 3, Zagreb HR-10000, Croatia.

E-mail: tudor@cirus.dhz.hr weaknesses of the LAM forecast caused by the LBCs was provided by Warner et al. (1997).

LBCs are obtained from models with a coarser mesh in the horizontal and the vertical that usually use simpler (different) parameterizations of physical processes. The coarse grid of the host model smooths the information supplied at the lateral boundaries (Caian and Geleyn 1997). The numerical procedures used on the interface of the two grids also generate errors (McDonald 1999). Termonia et al. (2009) showed that commonly used temporally interpolating lateral-boundary data may lead to errors in the surface field of up to $\sim 10 \mathrm{hPa}$ in case of fast propagating storms.

Model error due to LBCs can be significant since it propagates into the domain interior during the forecast (Nicolis 2007). It propagates and amplifies as it enters the domain of integration depending on the intensity of the cross-boundary flow and spreads farther through the domain with longer time of integration (Nutter et al. 2004). This problem is becoming more important as LAM forecasts tend to be longer, up to $72 \mathrm{~h}$ and in higher resolution, covering smaller area and with narrow coupling zone. Enlargement of the domain to move the edges far 
from the area of interest does not prevent the LBC error from eventually contaminating the solution (Vannitsem and Chomé 2005).

Juang and Kanamitsu (1994) developed a regional spectral model that predicts deviations from the global model forecast and find that shorter coupling intervals allow more noise in the mean sea level pressure field along lateral boundaries, but not in the precipitation field. To force the perturbations to zero along lateral boundaries and reduce the aforementioned noise, they apply lateral boundary relaxation for the dynamical part of the total tendency and a blending of the total tendency over the entire regional model domain. The second procedure was found unnecessary for noise removal. The subsequent study (Juang and Hong 2001), using the same model, revealed that it is not necessary to have a large domain in order to avoid lateral boundary influence and multinesting is not necessary for a very fine resolution forecast over a small domain. Assignment of lateral boundary values at the boundaries is found essential for representing scales too large to be periodic on LAM domains (Laprise 2003), which represents a largescale closure.

The schemes for lateral boundary conditions used in NWP usually specify every field at all the lateral boundaries making the initial-boundary problem mathematically ill posed (McDonald 1999). Unfortunately, Oliger and Sundström (1978) found that local pointwise boundary conditions cannot be well posed for hydrostatic equations and open boundaries. There are solutions in simplified models (see, e.g., McDonald 2000; Termonia and Voitus 2008) that allow well posedness and control the gravity waves, but the extension of the gravity wave control mechanism from one to more dimensions leads to fundamental difficulties (Durran 1999). The search for the well-posed solution continued [e.g., for the problem in semi-Lagrangian models when the origin point of the trajectory lies outside of the model domain (McDonald 2000)], the application of this work in spectral models (Termonia and Voitus 2008; Voitus et al. 2009) and improved schemes for overspecified LBCs (e.g., Navon et al. 2004). Spurious gravity waves that occur due to the ill posedness of the LBCs are filtered by the coupling procedure itself and/or the horizontal diffusion scheme and it is supposed that the remaining spurious waves are acceptable. Even when the problem is well posed, waves can still be reflected from the boundary. Boundaries that transmit waves in and out without spurious reflections are said to be transparent (McDonald 2002). Such a set has been tested in a nested environment on a simple set of shallow-water equations (McDonald 2003) on a single level without diffusive terms. However, the results still depend on the quality of the large-scale data used for coupling.
The quality of the LBC data for operational as well as research purposes is severely restricted since its amount is limited by storage and data transfer capacities. Largescale fields are usually available in temporal resolution of several hours, but they are needed at each LAM time step, which is usually on the order of several minutes. Consequently, LBCs are obtained at every LAM time step using large-scale fields that are interpolated in time. This interpolation procedure corrupts the fields, especially the features that have time scales shorter than the coupling interval. The situation can be made even worse when the large-scale fields are taken only from the narrow area close to the domain lateral boundaries. Consequently, small-scale features that are quick enough to enter the domain during one coupling interval are not suitably represented by the interpolated data (see Termonia 2003).

In Termonia (2004) it is shown that it is possible to detect boundary errors coming from such deficiencies in the interpolation. Termonia et al. (2009) proposed a solution that relies on a restart of the forecast after the storm has entered the domain and the error is detected by the boundary error procedure. This proposal improves the forecast itself but still exhibits two weaknesses that may be subject for improvements. The first is that a standard initialization like the popular digitalfiltering initialization (DFI) may weaken the depths of the large-scale storms present in the data of the coupling model. This can be controlled by using a scale-selective digital filter (SSDFI) as proposed in Termonia (2008). Second, any small-scale information that has been built up in the limited-area model since the beginning of the forecast run is lost. In that paper it is also suggested that this method may be improved in spectral models by relying on spectral nudging of the type proposed in Waldron et al. (1996), von Storch et al. (2000), Radu et al. (2008), and Guidard and Fischer (2008). In those papers the spectrally nudged information was used over the entire domain. Possible benefits of spectral nudging have been noticed by Meinke et al. (2006). The present paper makes a first feasibility study of such methods to improve the LBC temporal resolution problem, in particular investigating its use within the buffer zone at the lateral boundary of the domain only. As a comparison the spectral nudging over the entire domain will also be included in the present paper.

The aim here is to develop a simple coupling procedure that could be used operationally as a supplement or as an alternative to the flow-relaxation one either always, or when the quality of the LBCs is found insufficient by the monitoring procedure of Termonia (2004). Alternative time-interpolation schemes for LBC data are proposed. Different coupling procedures are implemented and tested using a simple one-dimensional model. 
This enables the identification of the errors linked to a particular LBC schemes, which could hardly be identified using a realistic model (Robert and Yakimiw 1986).

This paper is organized as follows. Section 2 outlines the problem by discussing the time evolution of spectral coefficients produced by an operational run of a realistic three-dimensional LAM. The one-dimensional model used for the testing of the alternative formulations, is also briefly described in this section. Results obtained using the flow-relaxation scheme are presented in section 3. The method of spectral coupling is described in section 4. Section 5 describes the time interpolation done in spectral space in combination with the usual gridpoint coupling scheme. The final section of this paper brings discussion and conclusions.

\section{Data and experimental setup}

This section analyses spectral data of a forecast for the operational Aire Limitée Adaptation Dynamique Développement International (ALADIN) limited-area numerical weather prediction model developed and maintained by the ALADIN International Team (1997). The obtained results will then be used as a basis for proposing improved temporal interpolation schemes in sections 4 and 5 .

Figures 1a,b show the evolution of the mean sea level pressure (MSLP) of the Lothar storm (Wernli et al. 2002) in an operational forecast of the ALADIN model between 0900 and 1200 UTC 26 December 1999. This model was run with a resolution of $\Delta x=\Delta y=9.5 \mathrm{~km}$ and 300 grid points in the zonal and meridional directions and a time step of $\Delta t=300 \mathrm{~s}$. Figure $1 \mathrm{c}$ shows the MSLP in the middle of this time interval at 1030 UTC. When linearly interpolating this storm within the 3-h time interval between $t_{0}=0900$ UTC and $t_{1}=1200$ UTC,

$$
\mathcal{L} c(t)=\frac{t_{1}-t}{t_{1}-t_{0}} c\left(t_{0}\right)+\frac{t-t_{0}}{t_{1}-t_{0}} c\left(t_{1}\right) .
$$

One gets at $t=1030$ UTC not one, but a "dipole" of two depressions, as can be seen from Fig. 1d. In most operational applications such interpolated data is used as coupling data for the Davies scheme. If, for instance, the configuration in Fig. 1d happened in the fictitious Davies zone shown in Figs. 1c and 1d, some completely spurious information might enter the physical domain of interest.

ALADIN is a spectral model following the work of Haugen and Machenhauer (1993), so the Fourier components of the fields can be easily obtained. The spectral coefficients are computed on an extension of the physical domain of the limited-area model, where the fields on the extension zone are constructed in such a way as to make the fields periodic using splines. During a time step computation the spectral information is present at the beginning of the time step and during the inversion of the Helmholtz equation, as explained in Table II of Termonia and Hamdi (2007). It is our aim here to investigate whether the spectral information may be useful to improve the proposals made in Termonia et al. (2009).

Within the ALADIN model a fast Fourier transform is applied twice in the two spatial horizontal directions $I$ and $J$ of the gridpoint field $F_{I J}$ with gridpoint indices $I=0, \ldots, M-1$, and $J=0, \ldots, N-1$ by

$$
c_{K L}=\operatorname{FFT}\left(F_{I J}\right)_{K L} \frac{1}{M N} \sum_{I=0}^{M-1} \sum_{J=0}^{N-1} F_{I J} e^{-(2 \pi i / M) I K} e^{-(2 \pi i / N) J L},
$$

for the indices $K=-M / 2, \ldots, M / 2$ and $L=-N / 2, \ldots$, $N / 2$, corresponding to waves with wave lengths $l_{K L}=$ $\left[(K / M \Delta x)^{2}+(L / N \Delta y)^{2}\right]^{-1 / 2}$.

The spectral coefficients are available for each model time step in the interval $\left(t_{0}, t_{1}\right)$ :

$$
c_{K L}^{\alpha}=c_{K L}(\alpha \Delta t)
$$

for $\alpha=0, \ldots, n_{t}$ corresponding to $t=t_{0}+\alpha \Delta t$, with $\Delta t$ the model integration time step. It can be easily verified that applying $\mathcal{L}$ to the gridpoint field $F_{I J}$ is equivalent to applying it to the spectral coefficients $c_{K L}$ :

$$
\operatorname{FFT}\left(\mathcal{L} F_{I J}\right)_{K L}=\mathcal{L} c_{K L}
$$

so the effect of the linear interpolation in Eq. (1) can be studied by investigating its effect on each separate spectral coefficient.

Figure 2 shows the time evolution of the three coefficients $c_{11,-15}, c_{1,0}$, and $c_{18,3}$ for the surface pressure between time $t_{0}=9$ - and $t_{1}=12$-h forecast range of the forecast run presented in Fig. 1. It can be seen from the time evolution of $c_{11,-15}$ in Fig. $2 \mathrm{a}$ that even though the linear interpolation may be quite good in the middle of the interval (indicated by the diamonds), it can completely miss the rotating part of the time evolution of the spectral coefficient. So the interpolation should be considered in all points in the interpolation interval. Figure $2 \mathrm{~b}$ shows for the large scales (illustrated here by $c_{1,0}$ with a wavelength of $2850 \mathrm{~km}$ ) that the linear interpolation is a good approximation. On the other hand for the small scales, exemplified here by $c_{18,3}$ with wavelength $l_{18,3}=156 \mathrm{~km}$, the interpolation is entirely wrong.

The time evolution of the spectral coefficients $c_{K L}^{\alpha}$ in Fig. 2 can be seen as a superposition of a linear trend and a rotation in the complex plane: 

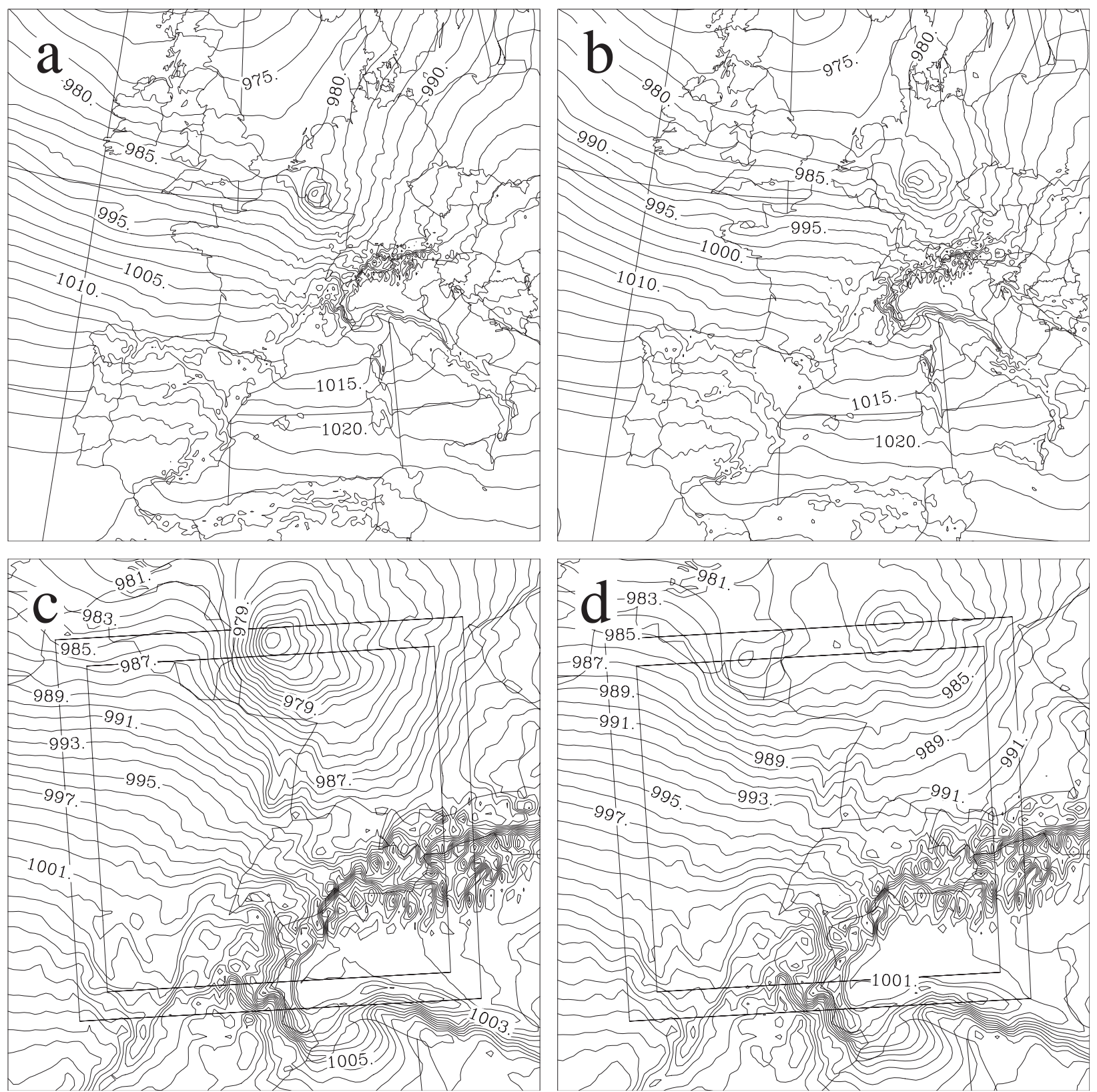

FIG. 1. ALADIN-France forecast of the Christmas storm on 26 Dec 1999: the MSLP at (a) 0900 UTC (contour interval is $2.5 \mathrm{hPa}$ ), (b) 1200 UTC (contour interval is $2.5 \mathrm{hPa}$ ), (c) 1030 UTC (zoom of the domain with contour interval of $1 \mathrm{hPa}$ ), and (d) the linear interpolation at 1030 UTC between the MSLP at 0900 UTC and the MSLP at 1200 UTC (zoom of the domain with contour interval of $1 \mathrm{hPa}$ ). The frame on (c) and (d) is a fictitious Davies relaxation zone containing the dipole structure of the interpolated field in (d).

$$
F_{K L}(t)=\Phi_{K L}(t)+\mathcal{A}_{K L}(t)
$$

with the linear trend given by

$$
\Phi_{K L}(t)=\Phi_{K L}\left(t_{0}\right)+\left(t-t_{0}\right) v_{K L},
$$

and the complex rotational part given by

$$
\mathcal{A}_{K L}(t)=M_{K L} e^{i\left[\Omega_{K L}\left(t-t_{0}\right)+\lambda_{K L}\right]} .
$$

The term $\Phi_{K L}$ can be interpreted as the part of the field that is locally growing (both positively or negatively) with tendency $v_{K L}$. The term $\mathcal{A}_{K L}$ represents the moving part of the wave.

Figure 3 shows some examples of the time evolution of selected spectral coefficients of the ALADIN forecast of the Christmas storm between 0900 and 1200 UTC to Eq. (5). Each time step is represented by a small rectangle. A fit of the function in Eqs. (5)-(7) is superposed 

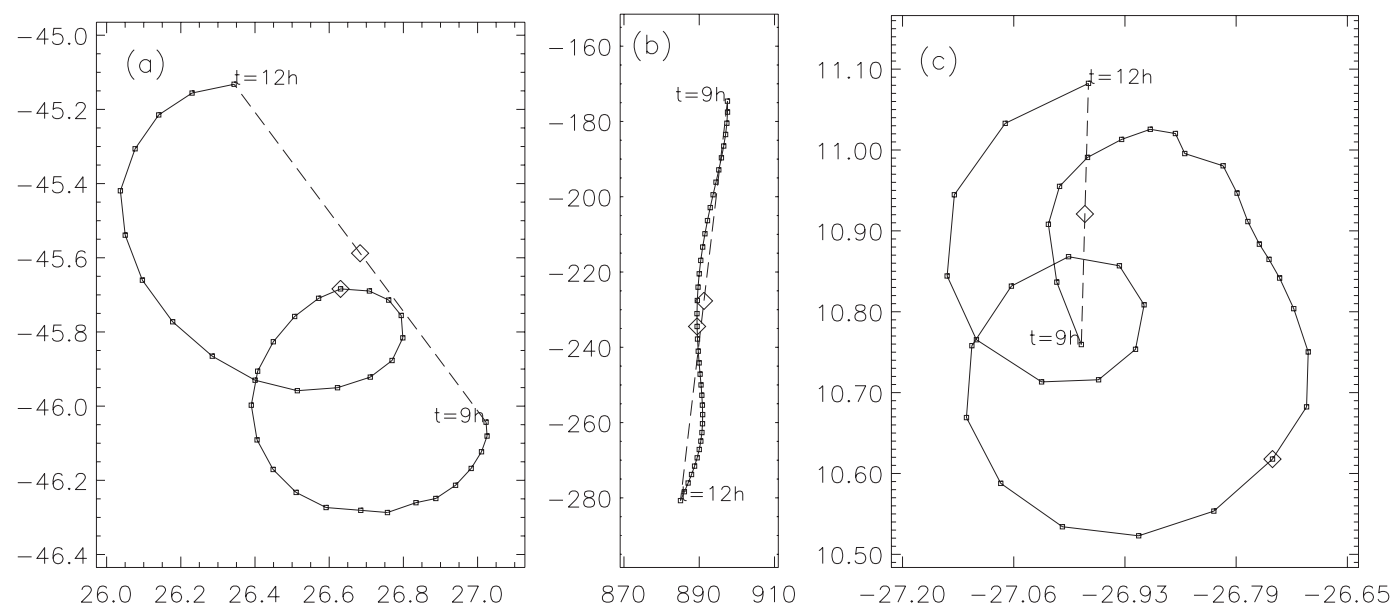

FIG. 2. Example of the time evolution of three spectral coefficients: (a) $c_{11,-15}$, (b) $c_{1,0}$, and (c) $c_{18,3}$. The $x$ axis and the $y$ axis indicate the real and the imaginary part, respectively, in units of $\mathrm{Pa}$.

in each panel (solid lines). ${ }^{1}$ This fit quantifies the validity of the hypothesis that the evolution can be decomposed into a rotating and a linear part.

From Fig. 3 we see that within time intervals of a few hours ( $3 \mathrm{~h}$ in this case) and for the large scales [i.e., the scales of the storm (100 km and more)], at the level of the spectral coefficients, the time evolution manifests itself as a combination of a linear trend and a rotation in the complex plane. Note that the fit is better for larger length scales. For instance in Figs. 3j, l corresponding to wave lengths $l_{16,-3}=175 \mathrm{~km}$ and $l_{19,19}=106 \mathrm{~km}$, the fits are of lower quality.

The aim of the present paper is to test whether this behavior of the spectral coefficients can be exploited to improve the LBC temporal resolution problem. As mentioned above this will be studied in a one-dimensional spectral shallow-water model on a single horizontal level. It uses velocity and geopotential as model fields and it can run on global or limited-area domains. The term global domain herein describes a periodic domain where a signal that exits on one end reenters on the opposite side. Use of the limited-area domain implies a coupling procedure on the domain edges. It is integrated with a two time level semi-implicit semi-Lagrangian scheme with a second-order accurate treatment of the nonlinear residual (Gospodinov et al. 2001).

A shallow-water spectral limited-area model that applies Fourier spectral representation on the model variables requires usage of time-dependent periodic LBCs

\footnotetext{
${ }^{1}$ The fit was taken as the optimal estimate for the parameters in $F_{K L}$ by minimizing the cost function $I\left(\Omega_{K L}, M_{K L}, \lambda_{K L}, v_{K L}, \Phi_{K L}\right)=$ $1 / 2 \sum_{\alpha=0}^{n_{t}} \overline{\left[F_{K L}\left(t_{0}+\alpha \Delta t\right)-c_{K L}^{\alpha}\right]}\left[F_{K L}\left(t_{0}+\alpha \Delta t\right)-c_{K L}^{\alpha}\right]$, by a conjugate gradient method (following Gilbert and Nocedal 1992). The bar denotes the complex conjugate.
}

(Haugen and Machenhauer 1993). Semi-implicit time integration and solving the Helmholtz equation in spectral space constrains the coupling procedure to be applied at the very beginning or end of the gridpoint computations (Radnóti 1995). Another solution would be to develop a simple and cheap procedure that can be applied in the spectral space. The width of the extension zone is determined by the fact that the extended boundary fields should be well represented by the used truncation (Haugen and Machenhauer 1993). The nonlinear terms of the model equations are computed without aliasing if the number of grid points in the whole integration area is chosen so that $N_{x}>3 M+1$ where $M$ is the truncation wavenumber. Weak numerical diffusion is applied in spectral space at the end of the time step to alleviate accumulation of energy at the smallest scales due to spectral blocking.

The large-scale model is a periodic low-resolution model that provides LBCs and will be referred to as the global model hereafter. In the tests, two sets of model runs are performed: the global and the LAM. The global and LAM models are using the same initial conditions that consist of a Gaussian shape depression that propagates from west to east with constant speed through the whole domain.

The global model is run on 200 grid points with $\Delta x=$ $40 \mathrm{~km}$ and the truncation wavenumber 66 . The LAM run is on 200 grid points: 11 of them are the extension zone on the east and the 8 points on the eastern and western edge of the remaining 189 points are the relaxation zones. The horizontal resolution of the LAM is $\Delta x=10 \mathrm{~km}$ and the truncation wavenumber is equal to the one used in the global model since the number of grid points is the same. Both models use the same time step of $150 \mathrm{~s}$. 

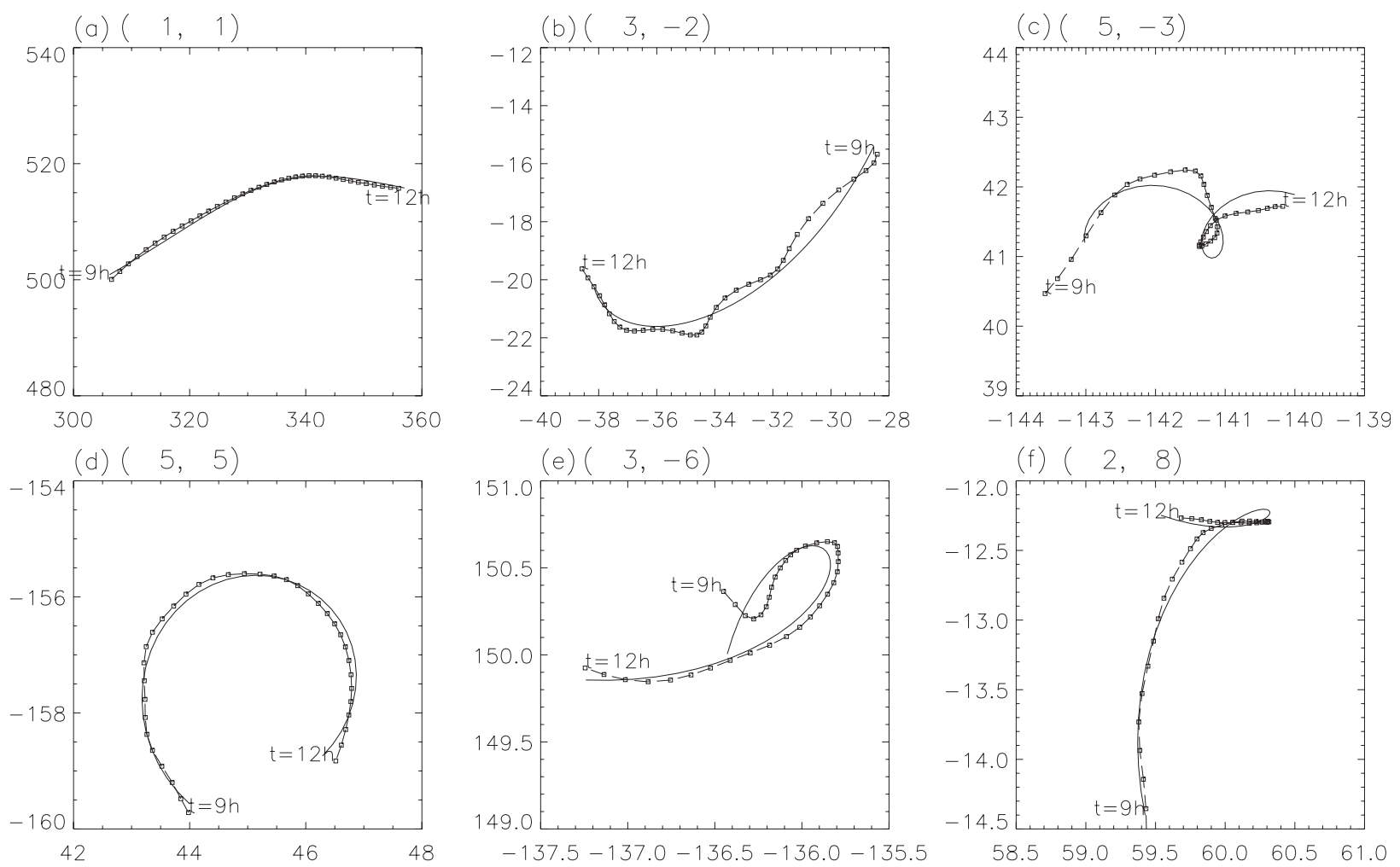

(g) $(8,3)$

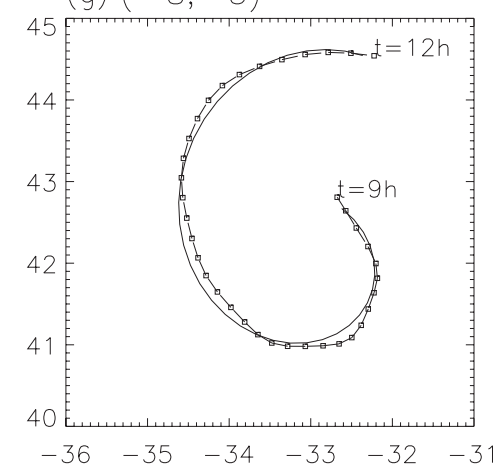

$-137.5-137.0-136.5-136.0-135.5$
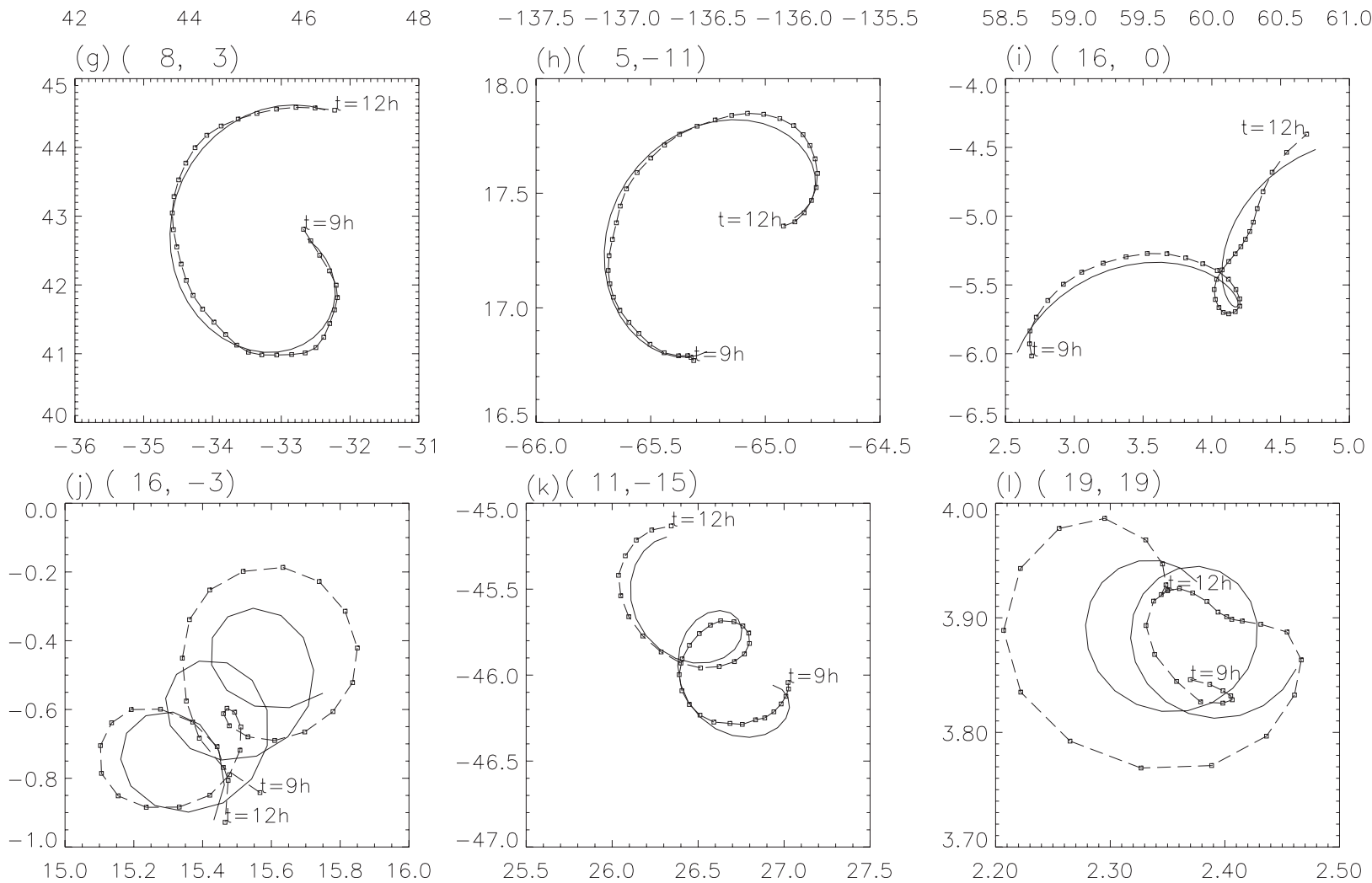

FIG. 3. Fits (solid lines) of selected spectral coefficients of the ALADIN forecast of the Christmas storm between 9-h and 12-h forecast range, compared to the forecast data: (a) $c_{1,1}^{\alpha}$, (b) $c_{3,-2}^{\alpha}$, (c) $c_{5,-3}^{\alpha}$, (d) $c_{5,5}^{\alpha}$, (e) $c_{3,-6}^{\alpha}$, (f) $c_{2,8}^{\alpha}$, (g) $c_{8,3}^{\alpha}$, (h) $c_{5,-11}^{\alpha}$, (i) $c_{16,0}^{\alpha}$, (j) $c_{16,-3}^{\alpha}$, (k) $c_{11,-15}^{\alpha}$, and (l) $c_{19,19}^{\alpha}$ (points). The $x$ and $y$ axis indicate the real and imaginary part, respectively, in Pa. 
Time steps when the large-scale data are available will be referred to as the coupling steps. They are separated by the coupling interval. The coupling procedure is done at each time step. It consists of spatial and temporal interpolation and the coupling scheme (e.g., the Davies 1976 scheme). The large-scale data are interpolated in space onto the LAM grid and then interpolated in time to be used at each LAM time step. The 3-h coupling interval is 72 time steps of the LAM.

\section{Gridpoint coupling}

This section demonstrates the capability of the simple model described in the previous section, to reproduce problems associated with interpolation of LBC in time on narrow lateral zones. The flow-relaxation coupling scheme proposed by Davies (1976) relaxes the interior flow to the prescribed exterior flow consuming gravity wave energy and fine spatial-scale potential vorticity in a narrow zone near lateral boundaries representing adequately the outgoing gravity waves as well as geostrophic flow through the boundary. This zone is called the relaxation zone and its width will be eight grid points of the LAM domain in the following tests. On the lateral boundaries, the LAM is forced with the large-scale solution. The value of the model variable in the relaxation zone $X_{C}$ is computed from the large-scale $\left(X_{\mathrm{LS}}\right)$ and the small-scale $\left(X_{\mathrm{SS}}\right)$ values by

$$
X_{C}=\alpha X_{\mathrm{LS}}+(1-\alpha) X_{\mathrm{SS}},
$$

using the relaxation coefficient $\alpha$ :

$$
\alpha=(p+1) Z^{p}-p Z^{p+1},
$$

where $p$ is the order of the polynomial (tuning parameter) and $Z=\left|x-x_{e}\right| /\left(x_{c}-x_{e}\right)$ is the distance of the grid point $x$ from the domain edge $x_{e}$ relative to the width of the coupling zone $\left(x_{c}-x_{e}\right)$. The relaxation coefficient $\alpha=1$ in the extension zone and $\alpha=0$ in the central zone of LAM.

The large-scale solution is known only at coupling steps $t_{0}, t_{1}, t_{2}, \ldots$, where $t_{0}$ is usually the initial time and the coupling intervals usually kept constant (e.g., in operational applications $3 \mathrm{~h}$ ), which is much longer than the typical time step used in operational LAM (5-10 min). The large-scale model state $X$ used in the relaxation zone is interpolated in time linearly:

$$
\begin{aligned}
X(t) & =w_{1} X_{t_{1}}+w_{2} X_{t_{2}} \\
\text { where } \quad w_{1} & =\frac{t_{2}-t}{t_{2}-t_{1}} \quad \text { and } \quad w_{2}=\frac{t-t_{1}}{t_{2}-t_{1}}
\end{aligned}
$$

or quadratically

$$
\begin{aligned}
X(t) & =w_{1} X_{t_{1}}+w_{2} X_{t_{2}}+w_{3} X_{t_{3}} \\
\text { where } \quad w_{1} & =\frac{\left(t_{2}-t\right)\left(t_{3}-t\right)}{\left(t_{2}-t_{1}\right)\left(t_{3}-t_{1}\right)}, \quad w_{2}=\frac{\left(t_{1}-t\right)\left(t_{3}-t\right)}{\left(t_{1}-t_{2}\right)\left(t_{3}-t_{2}\right)} \\
\text { and } \quad w_{3} & =\frac{\left(t_{1}-t\right)\left(t_{2}-t\right)}{\left(t_{1}-t_{3}\right)\left(t_{2}-t_{3}\right)},
\end{aligned}
$$

or using the tendency of the model state (Termonia 2003):

$$
\begin{aligned}
X(t)= & w_{1} X_{t_{1}}+w_{2} X_{t_{2}}-w_{1} w_{2}\left(t_{2}-t_{1}\right) \\
& \times\left[(\partial X / \partial t)_{t_{2}}-(\partial X / \partial t)_{t_{1}}\right]
\end{aligned}
$$

where $w_{1}$ and $w_{2}$ are computed as in linear interpolation scheme. Another solution can be to increase the size of the coupling zone to include the area where the depression appears at the coupling step.

We need to determine the appropriate reference simulation to be used as reference for computation of error introduced by the coupling or time interpolation scheme. The effectiveness of the boundary updating was first tested using the method of Baumhefner and Perkey (1982).

- Test 1: The global model was run using the same horizontal resolution as the LAM, on 800 grid points with $\Delta x=10 \mathrm{~km}$ and the truncation wavenumber of 264. The LAM was run on the same domain as usual, but coupled to the high-resolution global model using the flow relaxation scheme. In the first test, output from the high-resolution global model was used from every time step so interpolation in time or space was not needed.

- Test 2: In the second test, the output from the highresolution global model was taken with a 3-h interval and interpolated in time only.

- Test 3: In the third test the output from the lowresolution global model was used from every time step so the LBC data were interpolated in space only.

There was no difference between the global and the LAM solutions in the first test when the flow relaxation scheme was used, as was expected (McDonald 1999). The difference between the results from the first and the second test represents the error due to the temporal interpolation procedure. The difference between results of the first and the third test represents the error due to spatial interpolation (Fig. 4) and different global model resolutions. The results of the global model run with different spatial resolutions are different. Consequently, LAM is coupled to the different global model data and the error is large. In other words, the disturbance that enters the domain is different so the error is not only due to spatial interpolation, but it is still lower than the error due to temporal interpolation. This is why the result of 


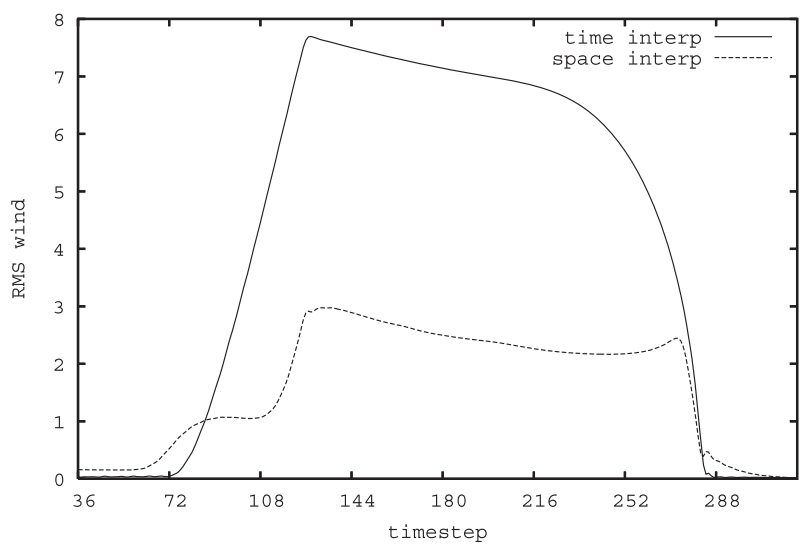

FIG. 4. RMSE of wind variable computed over the LAM domain using the LAM coupled to high-resolution global model as reference, for LAM coupled to high-resolution global data with 3-h interval (line) and coupled to low-resolution global data from every time step (dashed).

the third test will be used as reference in the rest of the article.

Using gridpoint coupling with large-scale data available with only 3-hourly interval allows for the depression to enter the domain area almost unnoticed (Fig. 5). When the same computational scheme is used but with new large-scale data available at every LAM time step (test 3 ), the disturbance is detected by the coupling scheme and further developed by the LAM (Fig. 6a). This result represents our ideal goal of "perfect coupling" to be reached by the modified or new coupling scheme. Unfortunately, such perfect conditions of data availability are hardly ever met by LAM users, so other options are tested. Quadratic interpolation in time does

(a)

Davies $3 \mathrm{~h}$ coupling $+03 \mathrm{~h}$

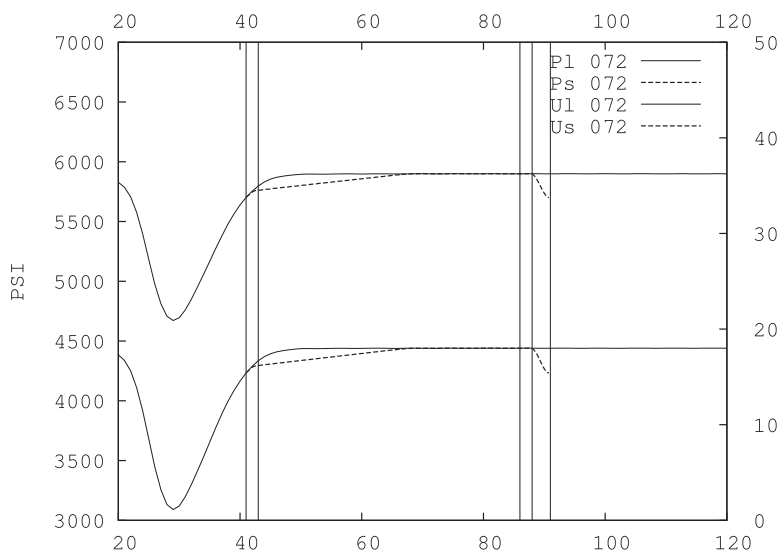

not improve the results (not shown) whereas using the tendencies as well as values of the model variables with 3-hourly interval does improve the results (Fig. 6b), but unfortunately, this is still far from the desired ideal. When the LAM domain was shifted so that the depression minimum enters the domain at the moment when the large-scale data are available, the depression was recognized, but its shape was distorted by the time interpolation of the large-scale data (Fig. 7a). Another simple geometry solution would be to increase the size of the coupling zone. When its width was fivefold its usual (Fig. 7b), the depression was recognized, but it also produced some spurious phenomena when the disturbance was leaving the domain.

Figure 8 shows the evolution of the model error due to the time-interpolation procedure (McDonald 1999) of the wind variable using test 3 as reference. The error increases as the disturbance enters the domain, between 72 and 144 time steps and decreases when it leaves the LAM domain, between 216 and 288 time steps. These last two results show that there is an error inherent in the temporal interpolation and/or the coupling scheme since it misinterprets or spoils the features that enter the domain, giving more incentive for finding an alternative coupling or more suitable time interpolation scheme.

\section{Spectral coupling}

As mentioned in the introduction, the coupling of LAM to a global model can be achieved using a procedure similar to spectral nudging that will be referred to as spectral coupling. This coupling is done over the whole domain area, not only the boundaries. The spectral

(b) Davies $3 \mathrm{~h}$ coupling $+06 \mathrm{~h}$

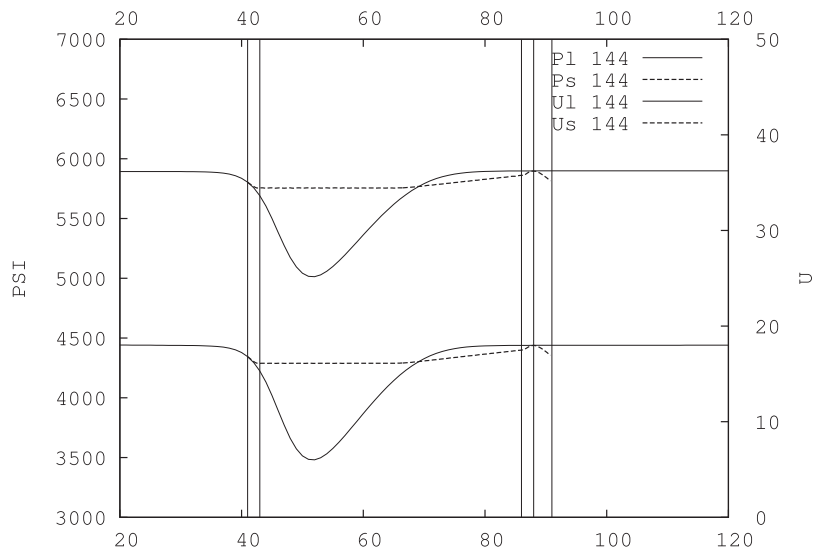

FIG. 5. Results for coupling using Davies scheme with 3-h interval between input large-scale data (a) before the depression enters the domain (3-h forecast) and (b) after (6-h forecast). Global model (solid line) and limited-area model (dashed) results for geopotential are shown above the results for the wind variable. (left to right) Vertical lines are left edge of the LAM domain, right edge of the left coupling zone, left edge of the right coupling zone, right edge of the right coupling zone (also left edge of the extension zone), and right edge of the LAM domain. 
(a)

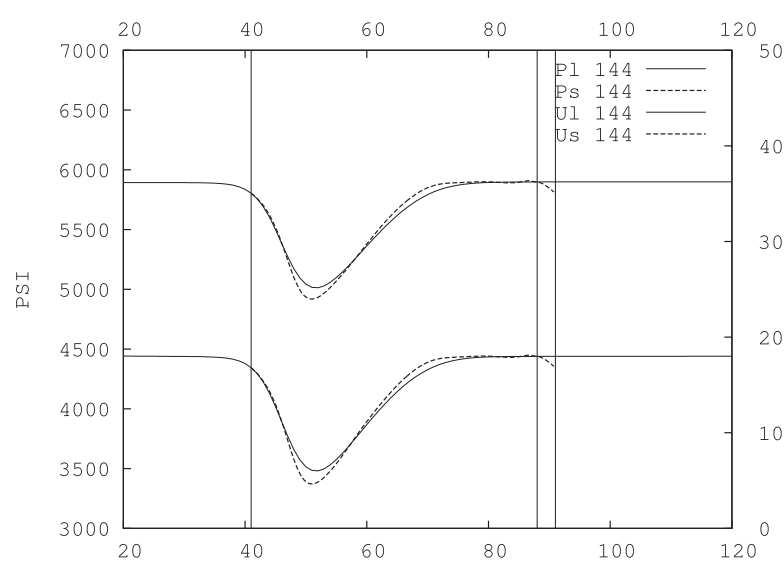

(b) Davies $3 \mathrm{~h}$ coupling $+06 \mathrm{~h}$

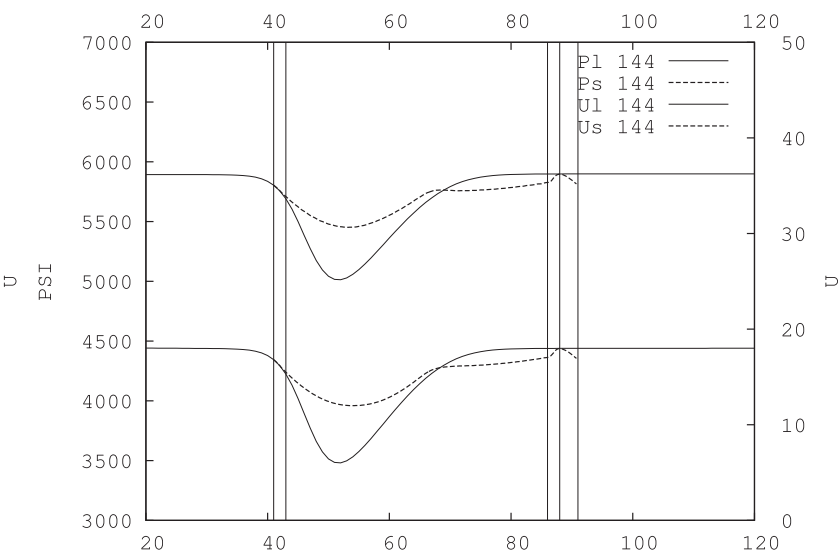

FIG. 6. Results for coupling using Davies scheme with (a) 1 time step interval between input large-scale data and (b) with 3-h interval between input large-scale data, but using tendencies of the large-scale fields for coupling. Both are 6-h forecasts. Lines have the same meaning as in Fig. 5.

coupling scheme was built using similar mechanism as the flow-relaxation scheme. The small wavenumber state (long waves) is taken from the large scale and the large wavenumber state (short waves) is taken from LAM with a smooth functional transition in between. In other words, the large-scale solution is spectrally filtered and blended with the LAM solution. The coupling scheme was developed on a basis of a spectral model used with a Fourier transform. The details are described in section 4a.

\section{a. The coupling method}

For wavenumbers lower than some threshold $k_{0}$ we take spectral coefficients from the large-scale model. For the wavenumbers larger than another threshold value $k_{1}$, the spectral coefficients are taken from the LAM. The spectral coefficients for wavenumbers between $k_{0}$ and $k_{1}$ are computed as

$$
\mathrm{SP}_{C}=\alpha \mathrm{SP}_{\mathrm{LS}}+(1-\alpha) \mathrm{SP}_{\mathrm{SS}}
$$

where the subscript $C$ denotes the coupled values, LS denotes the values from large-scale model, and SS denotes values from small-scale model. In analogy with the flow-relaxation scheme, the dependency of the $\alpha$ coefficient on the wavenumber $k$ can be linear

$$
\alpha=\frac{k_{1}-k}{k_{1}-k_{0}},
$$

(a)

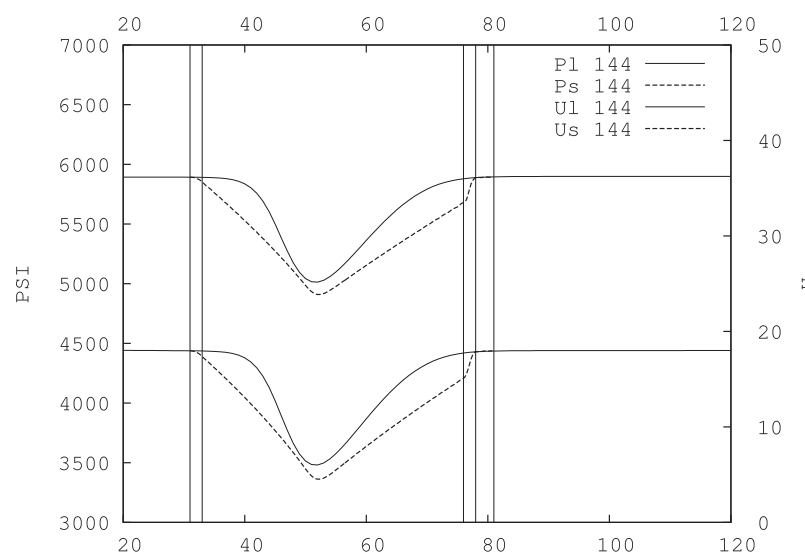

(b) Davies $3 \mathrm{~h}$ coupling $+06 \mathrm{~h}$

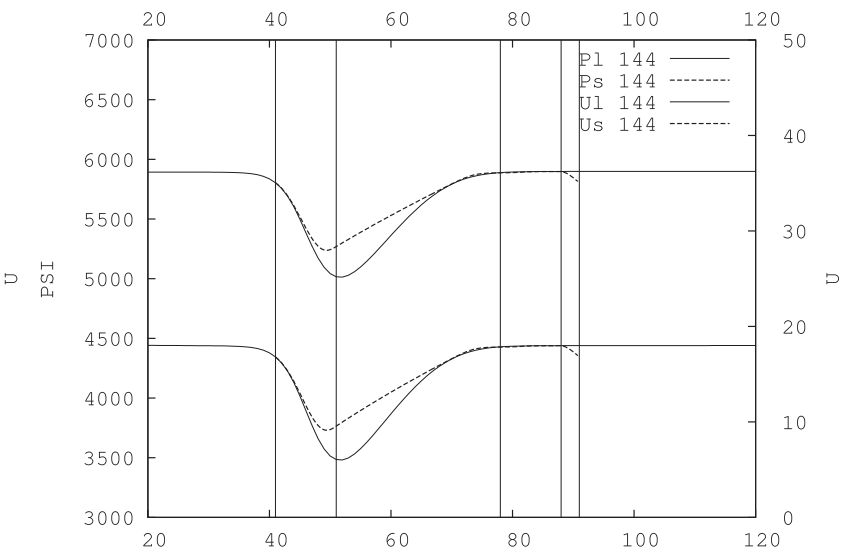

FIG. 7. Results for coupling using Davies scheme with 3-h interval between input large-scale data and LAM domain shifted so that the depression enters the domain (a) at the time the large-scale data are known and (b) when the coupling zone is increased 5 times: using 40 instead of 8 points. Lines have the same meaning as in Fig. 5. 


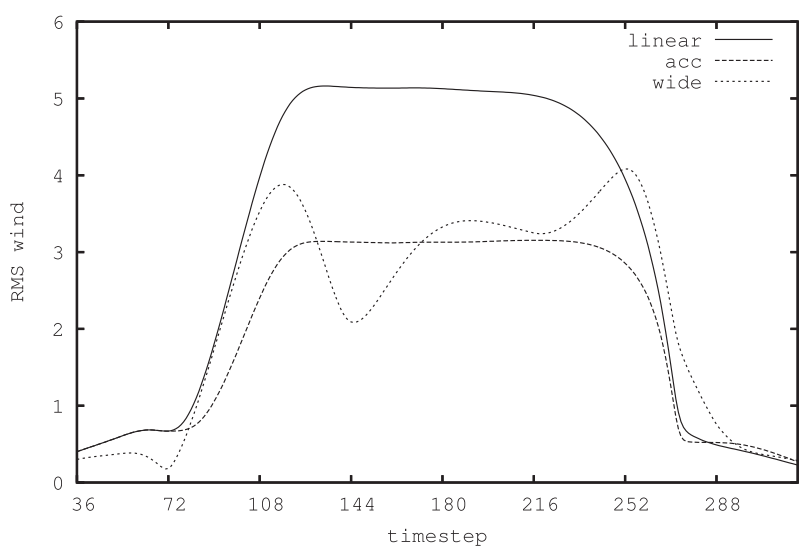

FIG. 8. RMSE of wind variable computed over the LAM domain using the LAM coupled to low-resolution global model for each time step as reference: for LAM coupled using flow relaxation scheme to low-resolution global data with 3-h interval interpolated linearly in time (line), using acceleration (long dash), and wider coupling area (short dash).

or have a polynomial dependence on $k$ :

$$
\begin{aligned}
& \alpha=(p+1) z^{p}-p z^{p+1} \quad \text { for } \quad p>0 \text { and } \\
& \alpha=1-(-p+1)(1-z)^{-p}-p z^{-p+1} \text { for } p<0
\end{aligned}
$$

where $z=\left(k_{1}-k\right) /\left(k_{1}-k_{0}\right)$ is the relative distance of the wavenumber $k$ from the small-scale wavenumber $k_{1}$ and $p$ is the order of the polynomial. The boundary wavenumbers $\left(k_{0}\right.$ and $\left.k_{1}\right)$ are tunable parameters, set according to the model resolutions and the size of the LAM domain. The choice of $k_{0}=2$ and $k_{1}=8$ address the need to describe the scales that are too large to be periodic in LAM (Laprise 2003) using lateral boundary data. The polynomial dependence of $\alpha$ on a wavenumber did not bring much improvement over the linear one in the tests using the simple one-dimensional model, so the linear dependence will be kept in the following experiments.

The spectral coupling scheme is scale selective, as the large scales are dominated by the spectra of the largescale model and only small scales are dominated by the spectra of the LAM. Its advantage is that the large-scale solution is forced to LAM on the whole domain area. Unfortunately, the spectral coupling scheme alone cannot eliminate spurious wave propagation from the lateral boundaries inward. Because of biperiodization, a necessity of a spectral LAM, without the gridpoint flow relaxation at the boundaries, all the waves that exit on one side of the domain freely enter on the opposite side. This is why we still need to use the gridpoint flow-relaxation scheme simultaneously to provide the damping on the domain edges. In other words, both coupling methods are combined. The relaxation takes place at the end of the gridpoint computations simultaneously with the flowrelaxation scheme.

\section{b. Coupling without interpolation of large-scale fields in time}

As shown in previous sections, time interpolation can introduce significant errors to the model results. These errors could be avoided by not doing the time interpolation at all. The large-scale fields are known only at discrete time intervals. In the gridpoint coupling scheme the coupling is done every time step and the large-scale fields on the boundaries are interpolated in time. Spectral coupling forces the large-scale solution LAM over the whole domain and could be done only at the coupling steps, when the large-scale data are available, or more often, up to every LAM time step.

First several options were tested of introducing largescale data into the LAM without being interpolated in time. The large-scale spectral coefficients are inserted to the LAM and the gridpoint part of the coupling scheme is left unchanged. If the LAM solution is forced by the large-scale one only at the coupling steps, the depression appears suddenly, during one time step. Such a result suggests that this method is not good for a real LAM with a more sophisticated dynamics and physics parameterization package.

Instead of introducing large-scale data suddenly, in one time step, an attempt was made to introduce it gradually during the coupling interval, so that coupling coefficient $\alpha$ was multiplied by a time-dependent $\beta$ function:

$$
\beta=\left\{\max \left[0, \frac{1}{1-t_{s}}\left(\frac{t-t_{1}}{t_{2}-t_{1}}-t_{s}\right)\right]\right\},
$$

where $t_{s}$ is the time when the large-scale solution from the second coupling time starts to be used, $t_{1}$ is the time of the first coupling file, and $t_{2}$ is the time of the second coupling file. The time $t$ is from the coupling interval $t_{1}<$ $t<t_{2}$. This way the large-scale data are not interpolated in time, but the data from the second coupling step are gradually introduced to the model during the coupling interval.

Unfortunately, such method leads to an unphysical solution of a false rapid generation of depression that develops in the domain, not an undisturbed transfer of a depression into the model domain. Therefore, we need to accomplish a different type of smooth transition between the coupling steps that would allow more physical representation of the model evolution on the lateral boundaries. 
(a)

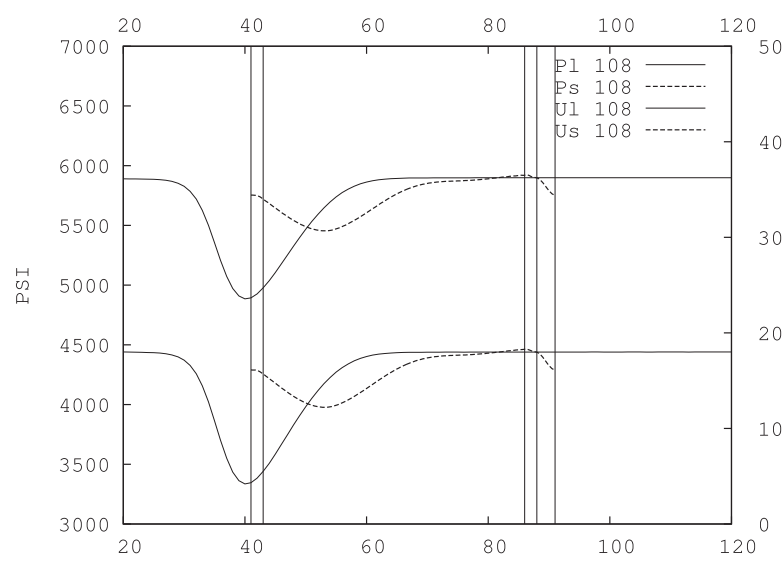

(b)

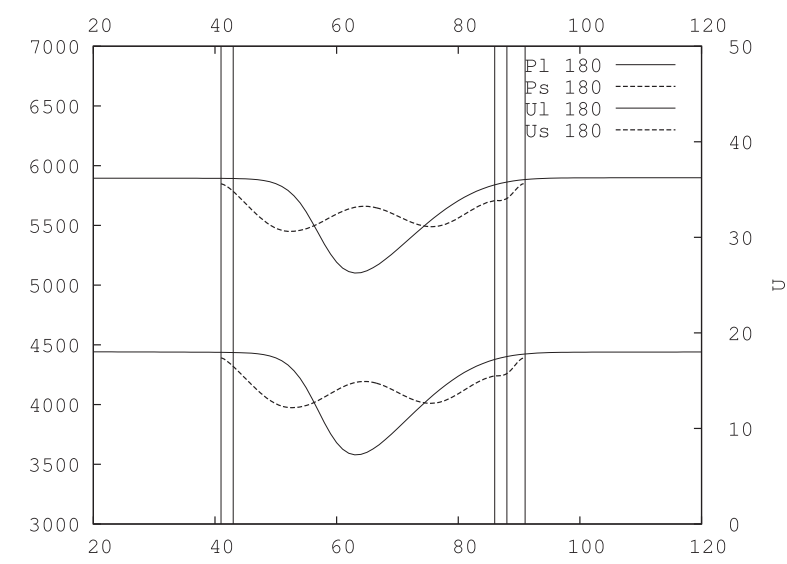

FIG. 9. Results using spectral coupling scheme with 3-h interval between input large-scale data, when the spectral coefficients are interpolated linearly in time after (a) 4.5 and (b) 7.5 h. Lines have the same meaning as in Fig. 5.

\section{c. Temporal interpolation of spectral coefficients}

The model uses spectral coefficients, so the first attempt was to use them in the time interpolation and avoid additional computations or transformations. The spectral coefficients of the large-scale fields are interpolated in time before being used by the coupling procedure. Regarding the spectral coefficients in a realistic LAM such as the ALADIN model, this corresponds to the assumption that they evolve in time linearly according to Eq. (6) and that the component in Eq. (7) is zero. This interpolation in time can be linear, but in analogy with the gridpoint coupling procedure above, a quadratic interpolation has also been investigated and the one that uses tendencies of the spectral coefficients. We use similar formulas as the ones in Eqs. (11) and (12) for gridpoint coupling when the values of the model fields are replaced by its spectral coefficients.

Results for linear interpolation of spectral coefficients in time are shown in Fig. 9. Instead of advection of the depression, a dipole is obtained. The depression develops and then dissolves only to develop on another position simultaneously. But even this unnatural model behavior led to improvements in the model error (see Fig. 12). Similar results were obtained for quadratic interpolation of spectral coefficients in time as well as when their tendencies (acceleration) were used. As shown in section 2, the time evolution of spectral coefficients is better represented with time interpolation of the linear trend and rotation in the complex plane. These can be seen as amplitude and phase of waves that constitute the field in spectral space. Since interpolation spectral coefficients in time also led to unrealistic model behavior, an attempt was made using amplitude and phase of spectral components.

\section{d. Temporal interpolation of amplitude and phase of spectral coefficients}

Amplitude and phase are first computed from the spectral components and then interpolated in time. The interpolated amplitude and phase are used to compute the large-scale spectral components used for coupling at a given time step. Linear and quadratic time interpolation of amplitude and phase is done using same the formulas as in gridpoint coupling schemes and acceleration is accounted for in an analogous way (Termonia 2003). This approach takes into account the fact that, in realistic LAMs such as the ALADIN model, also the phases corresponding to Eq. (7) evolve in time. The resulting model run showed significant improvements compared to the run when spectral coefficients were interpolated. The depression was mostly advected and the dipole problem almost disappeared. This result encouraged searching for alternative schemes for interpolation of amplitude and phase in time.

\section{1) Average of extrapolated values}

An alternative time interpolating scheme has been introduced that estimates the value of the model variable $X$ at time $t$ by extrapolating it from the coupling steps. Assume that model variable $X$ at one coupling step at time $t_{1}$ has known value $X_{1}$ and a time derivative $(\partial X / \partial t)_{t_{1}}$ and in the next coupling step at time $t_{2}$ has value $X_{2}$ and derivative $(\partial X / \partial t)_{t_{2}}$. The simplest way of accounting for the tendency in the interpolation scheme is to compute the forward extrapolated value from time $t_{1}$ :

$$
X_{1}(t)=X_{1}+(\partial X / \partial t)_{t_{1}}\left(t-t_{1}\right)
$$

and backward extrapolated value from time $t_{2}$ : 
(a)

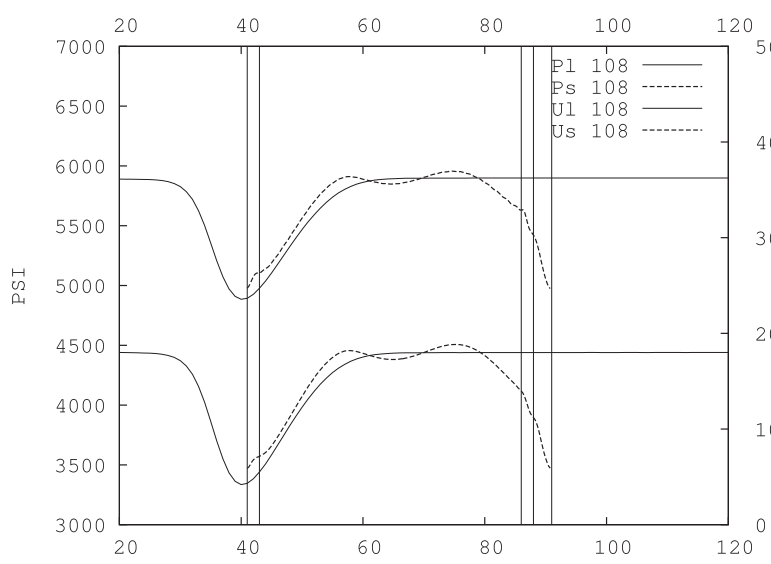

(b)

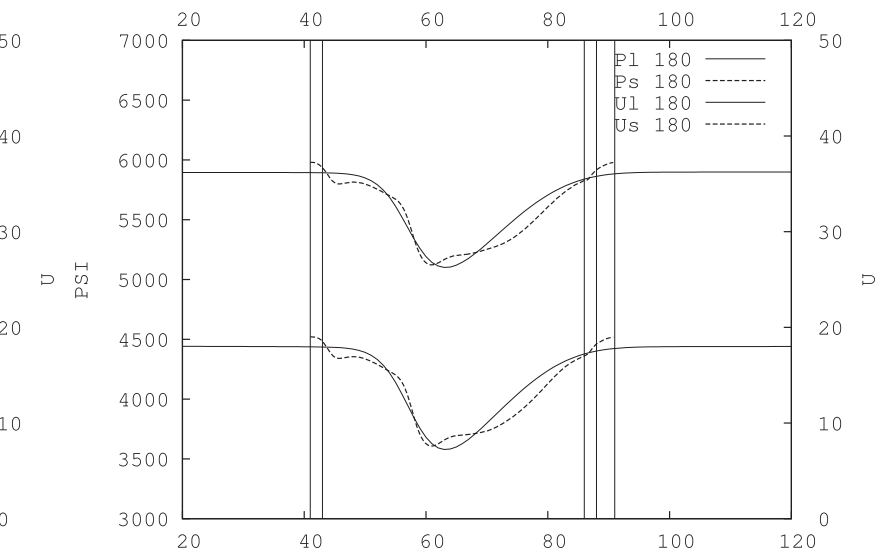

FIG. 10. Results using spectral coupling scheme with 3-h interval between input large-scale data, when amplitude and phase are interpolated in time using the extrapolated values after (a) 4.5 and (b) $7.5 \mathrm{~h}$. Lines have the same meaning as in Fig. 5.

$$
X_{2}(t)=X_{2}+(\partial X / \partial t)_{t_{2}}\left(t-t_{2}\right),
$$

and finally compute their weighted average:

$$
X(t)=w_{1} X_{1}(t)+w_{2} X_{2}(t),
$$

where $w_{1}$ and $w_{2}$ are the same as for the linear interpolation. Usage of this interpolating scheme allows the depression to smoothly enter the domain, to be advected through it, and then exit (Fig. 10). Unfortunately, there are a few spurious waves generated on top of the simulated depression that spoil the solution slightly. Another drawback is that the LAM contribution to the resulting model evolution is suppressed by the spectral nudging of the spectral components toward the large-scale solution. In other words, the LAM does not bring a useful contribution to the evolution of the model variables or this contribution is hidden with spurious waves that are consequence of the temporal interpolation of the large-scale fields.

\section{2) INTEGRATED WEIGHTED TENDENCIES}

Instead of using a fixed value for the tendency for the whole $\left(t-t_{1}\right)$ or $\left(t_{2}-t\right)$ period, we can use a weighted average of the two tendencies at each time step and then compute the integral from $t_{1}$ to $t$ or from $t$ to $t_{2}$, respectively.

The value of the model variable $X$ at time $t$ can be estimated by forward integration of the following expression:

$$
X_{1}(t)=X_{1}+\int_{t_{1}}^{t}\left[w_{1}(\partial X / \partial t)_{t_{1}}+w_{2}(\partial X / \partial t)_{t_{2}}\right] d t,
$$

where $w_{1}=\left(t_{2}-t\right) /\left(t_{2}-t_{1}\right)$ and $w_{2}=\left(t-t_{1}\right) /\left(t_{2}-t_{1}\right)$ are functions of time $t$. The obtained function of time is

$$
\begin{aligned}
X_{1}(t)= & X_{1}+(\partial X / \partial t)_{t_{1}}\left(t-t_{1}\right) \\
& +\frac{1}{2}\left[(\partial X / \partial t)_{t_{2}}-(\partial X / \partial t)_{t_{1}}\right] \frac{\left(t-t_{1}\right)^{2}}{t_{2}-t_{1}}
\end{aligned}
$$

or alternatively, a similar expression can be obtained when integrating from time $t_{2}$ backward:

$$
X_{2}(t)=X_{2}-\int_{t}^{t_{2}}\left[w_{1}(\partial X / \partial t)_{t_{1}}+w_{2}(\partial X / \partial t)_{t_{2}}\right] d t,
$$

yielding an alternative function of time:

$$
\begin{aligned}
X_{2}(t)= & X_{2}-(\partial X / \partial t)_{t_{2}}\left(t_{2}-t\right) \\
& +\frac{1}{2}\left[(\partial X / \partial t)_{t_{2}}-(\partial X / \partial t)_{t_{1}}\right] \frac{\left(t_{2}-t\right)^{2}}{t_{2}-t_{1}} .
\end{aligned}
$$

The final interpolation function is the linear combination of the two:

$$
X(t)=w_{1} X_{1}(t)+w_{2} X_{2}(t) .
$$

This interpolation scheme generates far less spurious waves (Fig. 11) and apparently there is some benefit of the higher-resolution LAM run since it contributes to the evolution of the disturbance.

\section{3) Polynomial interpolation}

Another interpolation function can be computed using the values of the model variable $X$ and its derivative at times $t_{1}$ and $t_{2}$ to evaluate coefficients in a third-order polynomial. First assume a polynomial dependence of the variable $X$ in time:

$$
X(t)=a+b t+c t^{2}+d t^{3}
$$


(a)

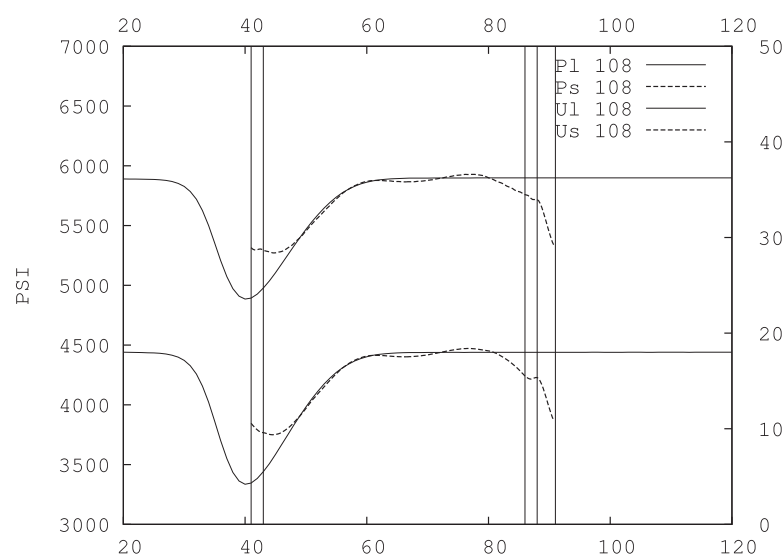

(b) Spectral $3 \mathrm{~h}$ coupling $+07.5 \mathrm{~h}$

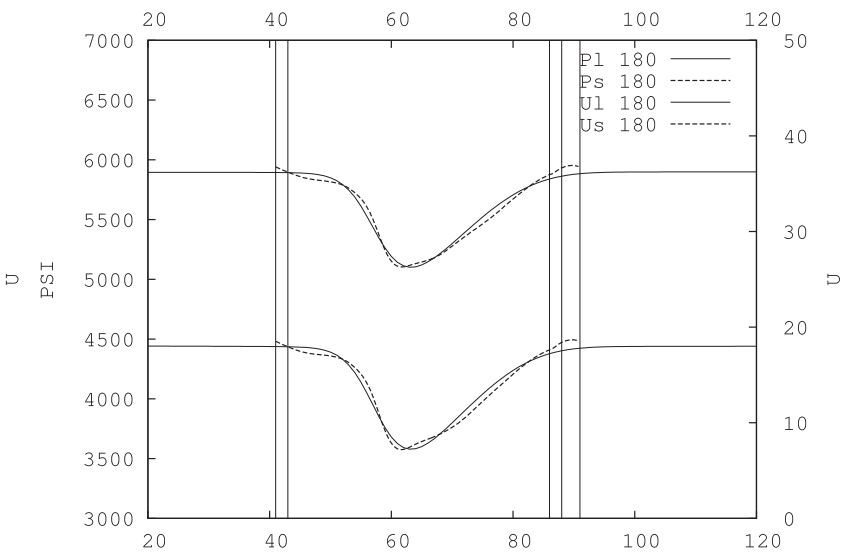

FIG. 11. Results using spectral coupling scheme with 3-h interval between input large-scale data, when amplitude and phase are interpolated in time using the integrated values after (a) 4.5 and (b) 7.5 h. Lines have the same meaning as in Fig. 5.

and compute the coefficients assuming $t_{1}=0$ for simplicity:

$$
\begin{aligned}
& a=X(t=0)=X_{1}, \\
& b=(\partial X / \partial t)_{t=0}=(\partial X / \partial t)_{t_{1}}, \\
& c=\frac{3}{t_{2}^{2}}\left\{X_{2}-X_{1}-\frac{1}{3}\left[2(\partial X / \partial t)_{t_{1}}+(\partial X / \partial t)_{t_{2}}\right] t_{2}\right\}, \quad \text { and } \\
& d=-\frac{2}{t_{2}^{3}}\left\{X_{2}-X_{1}-\left[(\partial X / \partial t)_{t_{1}}+(\partial X / \partial t)_{t_{2}}\right] t_{2}\right\} .
\end{aligned}
$$

This interpolation scheme also allows for the depression to smoothly enter the domain, but unfortunately it also amplifies several wave components more than it should so spurious waves appear in the LAM solution (figure not shown, results qualitatively similar to those in Fig. 10).

The spectral coupling procedure using temporal interpolation of amplitude and phase instead of spectral coefficients has reproduced the model evolution in more physical way yielding results that are similar to the test with gridpoint coupling using large-scale data from each time step-the perfect coupling test (Fig. 6a). The spectral coupling alone allows for waves to reenter the domain upon exiting on the opposite side because of the biperiodization of the large-scale fields. Therefore, it still requires simultaneous usage of the gridpoint coupling procedure on the domain edges to filter the waves that would otherwise reenter the domain.

The model error evolution (Fig. 12) shows the minimum values at coupling steps and maxima in the time between, when the error of the interpolation in time is largest. This is consistent with results from Nutter et al. (2004), who found the largest errors in the boundary zone near the midpoint of the LBC update cycle. The results suggest that integrated weighted tendencies give the least spurious waves while allowing for the disturbance to enter and leave the LAM domain.

Unfortunately, the temporal interpolation scheme in combination with the spectral coupling procedure and biperiodization might generate spurious waves that could spoil the solution or mask the LAM contribution to the model evolution. It is also possible that these spurious waves are partly a consequence of double coupling on the domain edges where the spectral coupling procedure could push the model fields in a different way than the

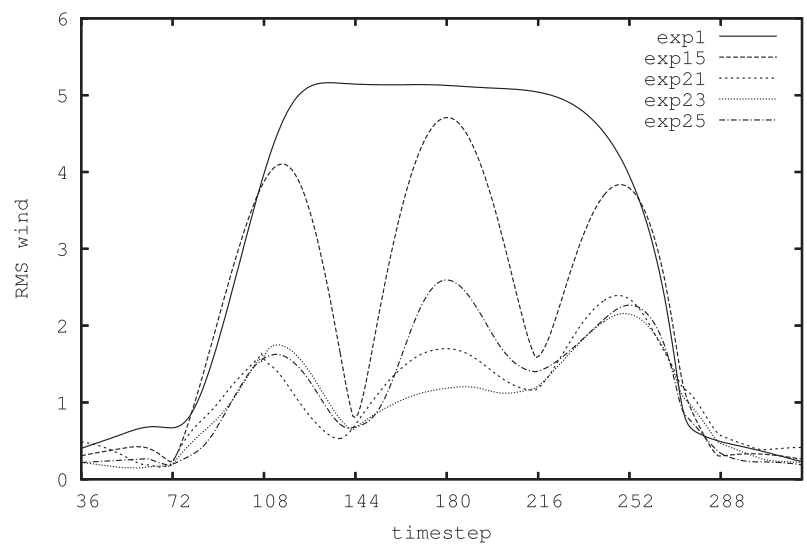

FIG. 12. RMSE of wind variable computed over the LAM domain using the LAM coupled to low-resolution global model for each time step as reference: for LAM coupled using flow-relaxation scheme to low-resolution global data with 3-h interval interpolated linearly in time (solid line), coupled using spectral coupling scheme when spectral coefficients are interpolated linearly in time (long dash), when the amplitude and phase of the spectral components are interpolated in time using extrapolation (short dash), and integration between coupling steps (dots) or polynomial interpolation in time (dot-dash). 
(a)

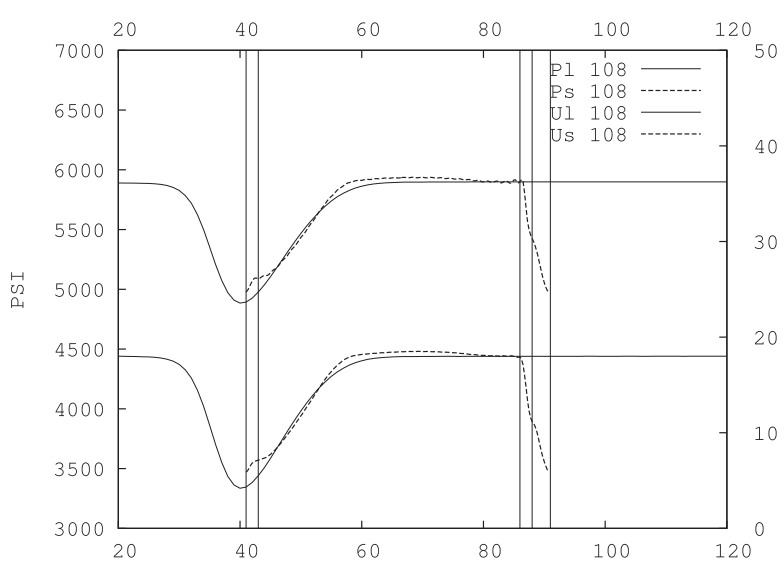

(b)

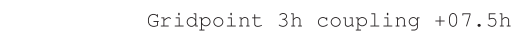

FIG. 13. Results using spectral coupling scheme with 3-h interval between input large-scale data when amplitude and phase are interpolated in time using the extrapolated values, but coupled in gridpoint space only in the narrow area close to the domain boundary, after (a) 4.5 and (b) 7.5 h. Lines have the same meaning as in Fig. 5.

gridpoint procedure. Therefore, another alternative is sought in the next section, which could potentially allow for physical evolution of LBC conditions and enable evolution of the LAM solution in the central part of the domain undisturbed by the spectral nudging toward the large-scale data.

\section{Gridpoint coupling using amplitude and phase angle interpolation in time}

The large-scale model state $X_{\mathrm{LS}}$ is transformed from the grid point to the spectral space, and the spectral coefficients are obtained. Then the amplitude and the phase angle of the complex spectral coefficients are computed and interpolated in time using the same procedures as when doing the spectral coupling. The time-interpolated amplitude and phase angle are used to compute the timeinterpolated spectral coefficients that are transformed back from spectral to gridpoint space. This way we obtain the large-scale fields used for gridpoint coupling.

The time interpolation of amplitude and phase can also be linear or quadratic, use acceleration, and have tendencies for integral or polynomial interpolation. When the amplitude and phase are interpolated linearly in time, the simulated depression is significantly weaker than with the perfect coupling scheme but can still be recognized. Unfortunately, when the depression leaves the domain, it is followed by a strong false positive signal (not shown). Results using quadratic coupling are very similar to the linear one. When the acceleration of amplitude and phase is used, the simulated depression is stronger and the false anticyclone is reduced. Using the average of extrapolated values gives satisfactory depth of the depression, but the amplitude of few short modes is a bit too strong (Fig. 13). Other results using tendencies of the model fields, either integrated between coupling steps or using polynomial interpolation give similar results as the simplest case shown in Fig. 13. The depression enters the domain, although it is less deep than in the large-scale model. But since this scheme relaxes the LAM solution to the large-scale one only in the narrow area close to the domain edge, the LAM can contribute to the development of the disturbance. Unfortunately, the other benefit of the gridpoint coupling is lost since the longest modes also reenter the domain, although much weaker. This is a consequence of the biperiodization of the large-scale fields. The evolution of the model error (Fig. 14) shows an increase after the depression leaves the domain due to these excessive waves.

\section{Discussion and conclusions}

The present paper aims to improve the LBC temporal resolution problem. A LAM that uses LBC data from a storage utility or remote center usually has the data available with a coupling interval of several hours. LBC data are interpolated in time and used in LAM each time step of several minutes. The features with time scales shorter than the coupling interval are corrupted or even removed by the time-interpolation procedure. The problem has encouraged the research on the coupling procedure that would enable a better representation of such features using the available LBC data.

It was shown (Fig. 1d) that linear interpolation of LBC within 3-h interval distorts the model fields. The interpolation procedure created two cyclones instead of one. The time evolution of the large-scale model fields is poorly represented by the time-interpolated fields on 


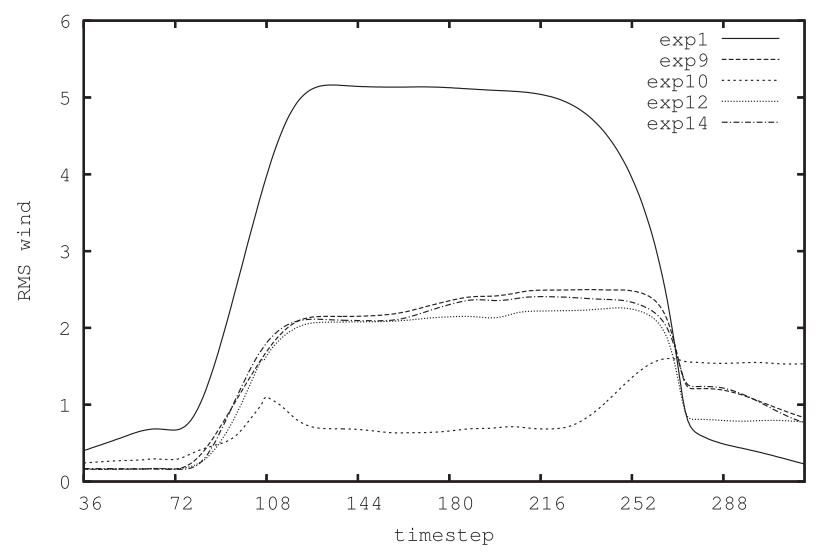

FIG. 14. RMSE of wind variable computed over the LAM domain using the LAM coupled to low-resolution global model for each time step as reference: for LAM coupled using flow-relaxation scheme to low-resolution global data with 3-h interval interpolated linearly in time (solid line), coupled in gridpoint space but the large-scale data are interpolated in spectral space, and when the amplitude and phase of the spectral components are interpolated in time using amplitude (long dash), extrapolation (short dash), integration between coupling steps (dots), or polynomial interpolation in time (dot-dash).

the domain edges. The evolution of model fields in time is better represented by a linear trend and a rotation of spectral coefficients in the complex plane (Fig. 4). This data obtained for a realistic 3D model served as inspiration to improve the temporal interpolation, in particular of the spectral coefficients. And these alternatives for the commonly used linear interpolation were tested using a simple $1 \mathrm{D}$ model. The tests reveal what error can be expected when using the different coupling and timeinterpolation schemes.

Gridpoint coupling, using standard Davies scheme on a narrow area close to the edges of the LAM domain with a coupling interval of several hours, misses a signal that enters the domain. Two possible alternatives to the standard Davies coupling are presented in the framework of a simple one-dimensional model. The first one does the coupling in the spectral space. This method is also known as spectral nudging and has shown benefits in other models (e.g., Meinke et al. 2006). The second one only interpolates the large-scale fields in time in spectral space but does the coupling in gridpoint space. Both of them are able to represent the missed signal in the LBC, but the second one could be the first step further from the "standard" gridpoint coupling using fields interpolated linearly in time.

Usage of the spectral coupling alone supports spurious waves that could reenter the domain as a consequence of biperiodization. These waves can be filtered by the gridpoint coupling scheme, as was done in previous studies when the boundary relaxation scheme was found necessary for LBC noise removal (Juang and Kanamitsu 1994).

Time interpolation in spectral space improves the representation of fast small-scale disturbances in LBC data. The LBC coupling scheme can benefit from the boundary-relaxation scheme used in combination with the improved time interpolation. Both schemes could be used either always or they could be applied only when the monitoring procedure proposed by Termonia (2004) detects that some signal has entered the LAM domain without being properly sampled by the standard 3-h linear temporal interpolation.

Acknowledgments. The original idea of the "dipole" problem and the investigation of the cure by the phaseangle interpolation was suggested to us by Jean-François Geleyn. This work benefited from numerous useful discussions with him and Gabor Radnóti. The authors thank the two anonymous reviewers. The manuscript was very much improved by their many pertinent remarks and suggestions. The first author thanks the Croatian Ministry of Science who supported this research through Grant 004-1193086-3036 to the Croatian Meteorological and Hydrological Service.

\section{REFERENCES}

ALADIN International Team, 1997: The ALADIN project: Mesoscale modelling seen as a basic tool for weather forecasting and atmospheric research. WMO Bull., 46, 317-324.

Baumhefner, D., and D. Perkey, 1982: Evaluation of lateral boundary errors in a limited-area domain. Tellus, 34, 409-428.

Caian, M., and J.-F. Geleyn, 1997: Some limits to the variable-mesh solution and comparison with the nested-LAM solution. Quart. J. Roy. Meteor. Soc., 123, 743-766.

Davies, H., 1976: A lateral boundary formulation for multi-level prediction models. Quart. J. Roy. Meteor. Soc., 102, 405-418.

_ 1983: Limitations of some common lateral boundary schemes used in regional NWP models. Mon. Wea. Rev., 111, 1002-1012.

Durran, D. R., 1999: Numerical Methods for Wave Equations in Geophysical Fluid Dynamics. Springer-Verlag, 465 pp.

Gilbert, J. C., and J. Nocedal, 1992: Global convergence properties of conjugate gradient methods for optimization. SIAM J. Optim., 2, 21-42.

Gospodinov, I., V. Spiridonov, and J.-F. Geleyn, 2001: Secondorder accuracy of two-time-level semi-Lagrangian schemes. Quart. J. Roy. Meteor. Soc., 127, 1017-1033.

Guidard, V., and C. Fischer, 2008: Introducing the coupling information in a limited-area variational assimilation. Quart. J. Roy. Meteor. Soc., 134, 723-735.

Haugen, J., and B. Machenhauer, 1993: A spectral limited-area formulation with time-dependent boundary conditions applied to the shallow-water equations. Mon. Wea. Rev., 121, 26182630.

Juang, H.-M., and M. Kanamitsu, 1994: The NMC nested regional spectral model. Mon. Wea. Rev., 122, 3-26.

- and S.-Y. Hong, 2001: Sensitivity of the NCEP regional spectral model to domain size and nesting strategy. Mon. Wea. Rev., 129, 2904-2922. 
Laprise, R., 2003: Resolved scales and nonlinear interactions in limited-area models. J. Atmos. Sci., 60, 768-779.

McDonald, A., 1999: A review of lateral boundary conditions for limited-area forecast models. Proc. Indian Nat. Sci. Acad., 65, 91-105.

— , 2000: Boundary conditions for semi-Lagrangian schemes: Testing some alternatives in one-dimensional models. Mon. Wea. Rev., 128, 4084-4096.

— 2002: A step toward transparent boundary conditions for meteorological models. Mon. Wea. Rev., 130, 140-151.

_- 2003: Transparent boundary conditions for the shallow-water equations: Testing in a nested environment. Mon. Wea. Rev., 131, 698-705.

Meinke, I., B. Geyer, F. Feser, and H. von Storch, 2006: The impact of spectral nudging on cloud simulation with a regional atmospheric model. J. Atmos. Oceanic Technol., 23, 815-824.

Navon, I. M., B. Neta, and M. Y. Hussiani, 2004: A perfectly matched layer approach to the linearized shallow-water equations model. Mon. Wea. Rev., 132, 1369-1378.

Nicolis, C., 2007: Dynamics of model error: The role of the boundary conditions. J. Atmos. Sci., 64, 204-215.

Nutter, P., D. Stensrud, and M. Xue, 2004: Effects of coarsely resolved and temporally interpolated lateral boundary conditions on the dispersion of limited-area ensemble forecasts. Mon. Wea. Rev., 132, 2358-2377.

Oliger, J., and A. Sundström, 1978: Theoretical and practical aspects of some initial boundary value problems in fluid dynamics. SIAM J. Appl. Math., 35, 419-446.

Radnóti, G., 1995: Comments on "A spectral limited-area formulation with time-dependent boundary conditions applied to the shallow-water equations." Mon. Wea. Rev., 123, 31223123.

Radu, R., M. Deque, and S. Somot, 2008: Spectral nudging in a spectral regional climate model. Tellus, 60A, 898-910.

Robert, A., and E. Yakimiw, 1986: Identification and elimination of an inflow boundary computational solution in limited area model integrations. Atmos.-Ocean, 24, 369-385.
Termonia, P., 2003: Monitoring and improving the temporal interpolation of lateral-boundary coupling data for limited area models. Mon. Wea. Rev., 131, 2450-2463.

_ 2004: Monitoring of the coupling update frequency of a limited-area model by means of a recursive digital filter. Mon. Wea. Rev., 132, 2130-2141.

_ 2008: Scale-selective digital filtering initialization. Mon. Wea. Rev., 136, 5246-5255.

_ - and R. Hamdi, 2007: Stability and accuracy of the physicsdynamics coupling in spectral models. Quart. J. Roy. Meteor. Soc., 133, 1589-1604.

— ditions from the dynamic core in semi-implicit semi-Lagrangian models. Tellus, 60A, 632-648.

—, A. Deckmyn, and R. Hamdi, 2009: Study of the lateral boundary condition temporal resolution problem and a proposed solution by means of boundary error restarts. Mon. Wea. Rev., 137, 3551-3566.

Vannitsem, S., and F. Chomé, 2005: One-way nested regional climate simulations and domain size. J. Climate, 18, 229-233.

Voitus, F., P. Termonia, and P. Bénard, 2009: Well-posed lateral boundary conditions for spectral semi-implicit semi-Lagrangian schemes: Tests in a one-dimensional model. Mon. Wea. Rev., 137, 315-330.

von Storch, H., H. Langenberg, and F. Feser, 2000: A spectral nudging technique for dynamical downscaling purposes. Mon. Wea. Rev., 128, 3664-3673.

Waldron, K. M., J. Peagle, and J. D. Horel, 1996: Sensitivity of a spectrally filtered and nudged limited area model to outer model options. Mon. Wea. Rev., 124, 529-547.

Warner, T., R. Peterson, and R. Treadon, 1997: A tutorial on lateral boundary conditions as a basic and potentially serious limitation to regional numerical weather prediction. Bull. Amer. Meteor. Soc., 78, 2599-2617.

Wernli, H., S. Dirren, M. A. Liniger, and M. Zillig, 2002: Dynamical aspects of the life cycle of the winter storm 'Lothar' (24-26 December 1999). Quart. J. Roy. Meteor. Soc., 128, 405-429. 